\title{
A Hamilton-Jacobi-Bellman approach for the numerical computation of probabilistic state constrained reachable sets
}

Mohamed Assellaou and Athena Picarelli

\begin{abstract}
Aim of this work is to characterise and compute the set of initial conditions for a system of controlled diffusion processes which allow to reach a terminal target satisfying pointwise state constraints with a given probability of success. Defining a suitable auxiliary optimal control problem, the characterization of this set is related to the solution of a particular Hamilton-Jacobi-Bellman equation. A semi-Lagrangian numerical scheme is defined and its convergence to the unique viscosity solution of the equation is proved. The validity of the proposed approach is then tested on some numerical examples.
\end{abstract}

\section{Introduction}

We consider the control of stochastic differential equations in $\mathbb{R}^{d}$ of the following form

$$
\left\{\begin{array}{l}
\mathrm{d} X(s)=b(s, X(s), u(s)) \mathrm{d} s+\sigma(s, X(s), u(s)) \mathrm{d} B(s), \quad \forall s \in[t, T] \\
X(t)=x
\end{array} .\right.
$$

Given a fixed time horizon $T>0$, we aim to characterize the set of initial states from which, with an assigned level of probability, it is possible to reach a target set at time $T$ satisfying some state constraints along the whole interval $[t, T]$.

More precisely, let $\mathscr{C}$ and $\mathscr{K}$ be two non-empty subsets of $\mathbb{R}^{d}$ representing respectively the target set and the set of state constraints and let $\rho \in[0,1)$. We define

Mohamed Assellaou

ENSTA ParisTech, 828 Boulevard des Marechaux, 91762 Palaiseau Cedex, France., e-mail: assellaou.med@gmail.com

Athena Picarelli

Mathematics Department, Imperial College. Huxley Building, 180 Queen's Gate, London SW72AZ, United Kingdom e-mail: a.picarelli@imperial.ac.uk 
the state constrained backward reachable set under probability of success $\rho$ as the set, hereafter denoted by $\Omega_{t}^{\rho}$, of initial points $x \in \mathbb{R}^{d}$ for which the probability to steer the system (1) towards $\mathscr{C}$ maintaining the dynamics in the set $\mathscr{K}$ is higher than $\rho$, i.e.

$$
\left\{x \in \mathbb{R}^{d}: \exists u \in \mathscr{U}, \mathbb{P}\left[X_{t, x}^{u}(\theta) \in \mathscr{K}, \forall \theta \in[t, T] \text { and } X_{t, x}^{u}(T) \in \mathscr{C}\right]>\rho\right\},
$$

where $X_{t, x}^{u}(\cdot)$ represents the strong solution to (1) associated with the control $u \in \mathscr{U}$. Assumptions on the coefficients in (1) and on the set of controls $\mathscr{U}$ will be made clear in the next section. Such backward reachable sets play an important role in many applications, as the set $\Omega_{t}^{\rho}$ can be interpreted as a "safety region" for reaching $\mathscr{C}$ remaining in the set $\mathscr{K}$, with confidence $\rho$. It turns out that the set $\Omega_{t}^{\rho}$ can be characterized by means of the so-called level set approach. At the basis of this approach there is the idea to look at the set of interest, the set $\Omega_{t}^{\rho}$ in our case, as the level set of a certain function solution of a suitable partial differential equation (PDE). Such a characterization of the set is particularly useful in view of its numerical approximation, since it opens the way to the use of a wide choice of numerical methods designed for PDEs. Originally introduced in [25] to model front propagation problems, this approach immediately resulted in a very powerful method for studying backward reachable sets of continuous non-linear dynamical systems under very general conditions. In $[16,24]$ this idea is used to describe the reachable sets for deterministic problems. The link between stochastic target problems and level set approach is established in [26]. More recently, the level set approach has been extended to the case of state-constrained controlled systems $[9,10]$ and probabilistic reachability problems [6].

In our case, we will show that it is straightforward to see that

$$
\Omega_{t}^{\rho}=\left\{x \in \mathbb{R}^{d}: \vartheta(t, x)>\rho\right\}
$$

where $\vartheta$ is the value function associated to the following optimal control problem:

$$
\vartheta(t, x):=\sup _{u \in \mathscr{U}} \mathbb{E}\left[\mathbb{1}_{\mathscr{C}}\left(X_{t, x}^{u}(T)\right) \bigwedge \min _{\theta \in[t, T]} \mathbb{1}_{\mathscr{K}}\left(X_{t, x}^{u}(\theta)\right)\right],
$$

with the standard notation $a \wedge b:=\min (a, b)$. In particular, equality (2) characterises the set $\Omega_{t}^{\rho}$ for $t \in[0, T]$ by means of the function $\vartheta$.

We point out that, in the discrete time setting, a similar approach has been considered in $[1,2,22]$. In this case, the value function is obtained recursively by solving the dynamic programming principle. In the present paper, we are interested in the approximation of the probabilistic backward reachable sets for time-continuous stochastic processes by PDE techniques. In the non controlled framework, an alternative numerical algorithm consist in using Monte Carlo simulations to generate a set of trajectories starting from a given initial position. The percentage of trajectories reaching the target without violation of the state constraints gives an approximation 
of the probability of success when starting from this position. On the other hand, for linear stochastic systems, a bound for the probability of hitting a target can be obtained by using the enclosing hulls of the probability density function for time intervals, see $[3,4]$. However, it is worth noticing that these approaches are used to calculate the probabilities of success but do not allow to define the entire set of points that have the same given probability. In addition, Monte-Carlo based methods often require a large number of simulations to obtain a good accuracy. We will use such simualtions in Section 5 as a comparison to validate our approach. In the context of financial mathematics, the problem of characterizing the backward reachable set with a given probability was first introduced by Föllmer and Leukert [18]. This problem was also studied and converted into the class of stochastic target problems by Touzi, Bouchard and Elie in [11]. However in these references the possible presence of state constraints is not taken into account.

In order to apply a dynamic programming approach and characterize the value function $\vartheta$ as the unique viscosity solution of a Hamilton-Jacobi-Bellman (HJB) equation we face two main difficulties. First, the discontinuous cost functional given by the presence of the indicator functions would require to make use of the notion of discontinuous viscosity solutions. Establish uniqueness results in such a framework is usually a very hard task, so we propose here to work on a regularized version of problem (3). Second, the non commutativity between expectation and maximum operator makes problem (3) not satisfying the natural "Markovian structure" necessary to apply the dynamic programming arguments. We here follow the ideas in $[8,10,19,21]$ and define an auxiliary optimal control problem in an augmented state space and derive the HJB equation for this problem recovering the value function $\vartheta$ solution of the original problem at a later stage. The obtained HJB equation is defined in a domain and completed with mixed Dirichlet and oblique derivative boundary conditions. Derivative conditions (to be considered in the viscosity sense, see Definition 1) typically arise dealing with running maxima in the cost functional (see also $[8,10]$ ), while the Dirichlet condition will be naturally satisfied pointwise by our value function. We discuss the numerical approximation of the obtained HJB equation. We introduce a semi-Lagrangian (SL) approximation scheme which incorporates the aforementioned boundary conditions and we prove its convergence to the viscosity solution following the framework in [7]. We recall that SL scheme for second order HJB equations have been introduced by Menaldi in [23] and then studied by Camilli and Falcone [13]. We refer to [15] and the references therein for an overview. Derivative boundary conditions have been added to the scheme in [10], while the case of mixed Dirichlet-derivative conditions has been recently studied in [21].

The paper is organised as follows. In Section 2 we present the problem and give some preliminary results. The regularized problem is introduced in Section 2.2. Section 3 is devoted to the development of the dynamic programming arguments and the HJB characterization. In Section 4 we discuss the numerical aspects and state the main convergence result. Numerical tests are presented in Section 5 


\section{Formulation of the problem and preliminary results}

\subsection{Problem formulation.}

Let $\left\{\Omega, \mathscr{F}_{t},\left\{\mathscr{F}_{t}\right\}_{t \geq 0}, \mathbb{P}\right\}$ be a filtered probability space and $B(\cdot)$ a given $p$-dimensional Brownian motion. Let $T>0$. We denote by $\mathscr{U}$ the set of all progressively measurable processes valued in $U \subset \mathbb{R}^{m}, U$ compact set. For any $u \in \mathscr{U}$, let us consider the following system of stochastic differencial equations (SDEs) in $\mathbb{R}^{d}$ :

$$
\left\{\begin{array}{l}
\mathrm{d} X(s)=b(s, X(s), u(s)) \mathrm{d} s+\sigma(s, X(s), u(s)) \mathrm{d} B(s), \quad \forall s \in[t, T] \\
X(t)=x
\end{array}\right.
$$

The following classical assumptions will be considered on the coefficients $b$ and $\sigma$ :

(Hb) : $[0, T] \times \mathbb{R}^{d} \times U \rightarrow \mathbb{R}^{d \times p}$ and $b:[0, T] \times \mathbb{R}^{d} \times U \rightarrow \mathbb{R}^{d}$ are continuous functions and there exist constants $L, M>0$ such that

$$
\begin{aligned}
& |b(t, x, u)-b(t, y, u)|+|\sigma(t, x, u)-\sigma(t, y, u)| \leq L(|x-y|), \\
& |b(t, x, u)|+|\sigma(t, x, u)| \leq M
\end{aligned}
$$

for any $t \in[0, T], x, y \in \mathbb{R}^{d}$ and $u \in U$.

It is well known that, under assumption (H1), for any $u \in \mathscr{U}$ there is a unique strong solution to (4) [27, p. 42, Thm. 6.3]. We denote by $X_{t, x}^{u}(\cdot)$ such a solution.

Let $\mathscr{C}$ and $\mathscr{K}$ be nonempty open sets in $\mathbb{R}^{d}$, representing respectively the target set and the set of state constraints. Let $\rho \in[0,1)$ an assigned value of success probability. We define the backward reachable set under probability of success $\rho$, as the set $\Omega_{t}^{\rho}$ of initial points $x \in \mathbb{R}^{d}$ from which it starts a trajectory $X_{t, x}^{u}(\cdot)$ such that the probability to reach the target $\mathscr{C}$ at the final instant $T$ satisfying the constraint $\mathscr{K}$ in the interval $[t, T]$ is grater than $\rho$, i.e.:

$\Omega_{t}^{\rho}:=\left\{x \in \mathbb{R}^{d}: \exists u \in \mathscr{U}, \mathbb{P}\left[X_{t, x}^{u}(\theta) \in \mathscr{K}, \forall \theta \in[t, T]\right.\right.$ and $\left.\left.X_{t, x}^{u}(T) \in \mathscr{C}\right]>\rho\right\}$.

For a given set $\mathscr{O} \subseteq \mathbb{R}^{d}$ we will denote by $\mathbb{1}_{\mathscr{O}}$ its indicator function, i.e.

$$
\mathbb{1}_{\mathscr{O}}(x):= \begin{cases}1 & \text { if } x \in \mathscr{O} \\ 0 & \text { otherwise. }\end{cases}
$$

One can easily verify that

$\mathbb{1}_{\mathscr{C}}\left(X_{t, x}^{u}(T)\right) \bigwedge_{\theta \in[t, T]} \min _{\mathscr{K}}\left(X_{t, x}^{u}(\theta)\right)= \begin{cases}1 & \text { if } X_{t, x}^{u}(\theta) \in \mathscr{K} \forall \theta \in[t, T] \text { and } X_{t, x}^{u}(T) \in \mathscr{C}_{(5)} \\ 0 & \text { otherwise, }\end{cases}$

i.e. the expression on the left hand side is an indicator function for the event 


$$
X_{t, x}^{u}(\theta) \in \mathscr{K} \forall \theta \in[t, T] \text { and } X_{t, x}^{u}(T) \in \mathscr{C} .
$$

It follows that, for any $u \in \mathscr{U}, \mathbb{P}\left[X_{t, x}^{u}(\theta) \in \mathscr{K}, \forall \theta \in[t, T]\right.$ and $\left.X_{t, x}^{u}(T) \in \mathscr{C}\right]$ can be expressed by

$$
\mathbb{E}\left[\mathbb{1}_{\mathscr{C}}\left(X_{t, x}^{u}(T)\right) \bigwedge \min _{\theta \in[t, T]} \mathbb{1}_{\mathscr{K}}\left(X_{t, x}^{u}(\theta)\right)\right] .
$$

As a consequence, it is possible to describe the set $\Omega_{t}^{\rho}$ using optimal control tools just looking at the evolution of the level sets of the following value function:

$$
\vartheta(t, x):=\sup _{u \in \mathscr{\mathscr { U }}} \mathbb{E}\left[\mathbb{1}_{\mathscr{C}}\left(X_{t, x}^{u}(T)\right) \bigwedge \min _{\theta \in[t, T]} \mathbb{1}_{\mathscr{K}}\left(X_{t, x}^{u}(\theta)\right)\right] .
$$

Proposition 1. Let assumption (H1) be satisfied. Then, for $t \in[0, T]$, we have:

$$
\Omega_{t}^{\rho}=\left\{x \in \mathbb{R}^{d}: \vartheta(t, x)>\rho\right\} .
$$

Proof. If $x \in \Omega_{t}^{\rho}$, thanks to equality (5)

$$
\mathbb{E}\left[\mathbb{1}_{\mathscr{C}}\left(X_{t, x}^{u}(T)\right) \bigwedge \min _{\theta \in[t, T]} \mathbb{1}_{\mathscr{K}}\left(X_{t, x}^{u}(\theta)\right)\right]>\rho
$$

for some control $u \in \mathscr{U}$ and it follows $\vartheta(t, x)>\rho$.

Let us now suppose that $\vartheta(t, x)>\rho$. By the definition of the supremum and the fact that $\mathscr{U}$ is a non empty set, one has that, for some control $\bar{u} \in \mathscr{U}$,

$$
\mathbb{E}\left[\mathbb{1}_{\mathscr{C}}\left(X_{t, x}^{\bar{u}}(T)\right) \bigwedge \min _{\theta \in[t, T]} \mathbb{1}_{\mathscr{K}}\left(X_{t, x}^{\bar{u}}(\theta)\right)\right]>\rho
$$

and then, using again (5), $x \in \Omega_{t}^{\rho}$.

Motivated by this result, we are going to focus on the characterization and numerical approximation of the function $\vartheta$. Problem (6) is an optimal control problem with a discontinuous cost in a "minimum form". This is not a standard formulation in optimal control theory for two main reasons: first, the discontinuity of the cost functional prevents the characterization of (6) as the unique viscosity solution of a HJB equation, second the loss of Markovian structure in the cost, due to the presence of the minimum operator inside the expectation, makes the dynamic programming arguments not directly applicable. We discuss the first issue in the next section.

\subsection{Regularized Problem}

The discontinuity introduced by the presence of the indicator functions and the consequent necessity of dealing with the notion of discontinuous viscosity solutions 
(see for instance [17, Section VII.4] for their definition) pose nontrivial issues when attempting to establish uniqueness results for the associated HJB equation. To overcome this difficulty, from now on we will work with a regularized version of the cost functional in (6). In particular, observing that the indicator functions $\mathbb{1}_{\mathscr{C}}$ and $\mathbb{1}_{\mathscr{K}}$ can be written as

$$
\mathbb{1}_{\mathscr{C}}(z)=\left\{\begin{array}{ll}
1 & \text { if } d_{\partial \mathscr{C}}(z)<0 \\
0 & \text { if } d_{\partial \mathscr{C}}(z) \geq 0
\end{array}, \quad \mathbb{1}_{\mathscr{K}}(z)= \begin{cases}1 & \text { if } d_{\partial \mathscr{K}}(z)<0 \\
0 & \text { if } d_{\partial \mathscr{K}}(z) \geq 0\end{cases}\right.
$$

where $d_{\partial \mathscr{C}}$ and $d_{\partial \mathscr{K}}$ are respectively the signed distance function to $\partial \mathscr{C}$ and $\partial \mathscr{K}$, we consider the following regularized functions $\phi_{\mathscr{C}}^{\mathcal{E}}$ and $\phi_{\mathscr{K}}^{\varepsilon}$ (see Figure 1):

$\phi_{\mathscr{C}}^{\varepsilon}(x):=\min \left(1, \max \left(0,-\frac{1}{\varepsilon} d_{\partial \mathscr{C}}(x)\right)\right), \quad \phi_{\mathscr{K}}^{\varepsilon}(x):=\min \left(1, \max \left(0,-\frac{1}{\varepsilon} d_{\partial \mathscr{K}}(x)\right)\right)$

and the optimal control problem

$$
\vartheta^{\varepsilon}(t, x):=\sup _{u \in \mathscr{U}} \mathbb{E}\left[\phi_{\mathscr{C}}^{\varepsilon}\left(X_{t, x}^{u}(T)\right) \bigwedge \min _{\theta \in[t, T]} \phi_{\mathscr{K}}^{\varepsilon}\left(X_{t, x}^{u}(\theta)\right)\right] .
$$

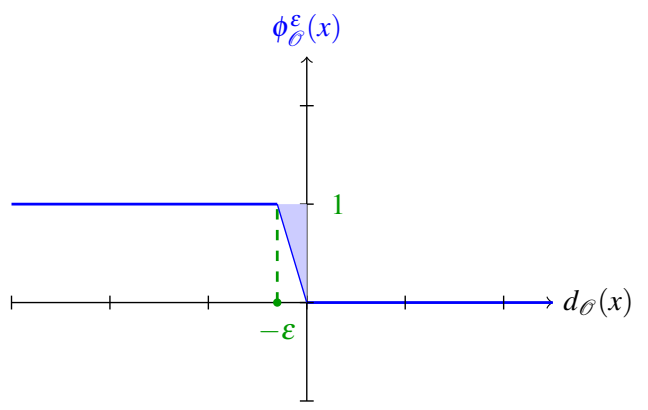

Fig. 1 Regularization of the indicator function in the case $\mathscr{O}=(-\infty, 0)$

Remark 1. Note that the choice of $\phi^{\varepsilon}$ is such that $\phi^{\varepsilon} \leq \mathbb{1}$. which implies

$$
\vartheta^{\varepsilon}(t, x)>\rho \Rightarrow \vartheta(t, x)>\rho .
$$

Hence if we are able to find a numerical approximation $\widetilde{\vartheta}^{\varepsilon}$ of $\vartheta^{\varepsilon}$ such that $\mid \widetilde{\vartheta}^{\varepsilon}-$ $\vartheta^{\varepsilon} \mid \leq \eta$ for some $\eta \geq 0$, we will have $\tilde{\vartheta}^{\varepsilon}(t, x)>\rho+\eta \Rightarrow \vartheta^{\varepsilon}(t, x)>\rho \Rightarrow \vartheta(t, x)>$ $\rho$.

This regularization allow us to deal with a continuous cost and also to obtain the following regularity result for the associated value function: 
Proposition 2. Let assumption (H1) be satisfied and let $\varepsilon>0$. The value function $\vartheta^{\varepsilon}$ is Lipschitz continuous with respect to $x$ and $\frac{1}{2}$-Hölder continuous with respect to $t$, i.e. there exists a constant $L_{\varepsilon}>0$ such that

$$
\left|\vartheta^{\varepsilon}(t, x)-\vartheta^{\varepsilon}(s, y)\right| \leq L_{\varepsilon}\left(|x-y|+|t-s|^{\frac{1}{2}}(1+|x|+|y|)\right)
$$

for any $t, s \in[0, T], x, y \in \mathbb{R}^{d}$.

Proof. Let $0 \leq t \leq s \leq T, x, y \in \mathbb{R}^{d}$. Thanks to the property of minimum operator $|(a \wedge b)-(c \wedge d)| \leq|a-c| \vee|b-d|$, one has:

$$
\begin{aligned}
& \left|\vartheta^{\varepsilon}(t, x)-\vartheta^{\varepsilon}(t, y)\right| \\
& \leq \sup _{u \in \mathscr{U}} \mathbb{E}\left[\left|\left(\phi_{\mathscr{C}}^{\varepsilon}\left(X_{t, x}^{u}(T)\right) \bigwedge \min _{\theta \in[t, T]} \phi_{\mathscr{K}}^{\varepsilon}\left(X_{t, x}^{u}(\theta)\right)\right)-\left(\phi_{\mathscr{C}}^{\varepsilon}\left(X_{t, y}^{u}(T)\right) \bigwedge \min _{\theta \in[t, T]} \phi_{\mathscr{K}}^{\varepsilon}\left(X_{t, y}^{u}(\theta)\right)\right)\right|\right] \\
& \leq \sup _{u \in \mathscr{U}} \mathbb{E}\left[\left|\phi_{\mathscr{C}}^{\varepsilon}\left(X_{t, x}^{u}(T)\right)-\phi_{\mathscr{C}}^{\varepsilon}\left(X_{t, y}^{u}(T)\right)\right| \bigvee \max _{\theta \in[t, T]}\left|\phi_{\mathscr{K}}^{\varepsilon}\left(X_{t, x}^{u}(\theta)\right)-\phi_{\mathscr{K}}^{\varepsilon}\left(X_{t, y}^{u}(\theta)\right)\right|\right]
\end{aligned}
$$

and

$$
\begin{aligned}
& \left|\vartheta^{\varepsilon}(t, x)-\vartheta^{\varepsilon}(s, x)\right| \\
& \leq \sup _{u \in \mathscr{U}} \mathbb{E}\left[\left|\phi_{\mathscr{C}}^{\varepsilon}\left(X_{s, X_{t, x}^{u}(s)}^{u}(T)\right)-\phi_{\mathscr{C}}^{\varepsilon}\left(X_{s, x}^{u}(T)\right)\right| \bigvee \max _{\theta \in[t, s]}\left|\phi_{\mathscr{K}}^{\varepsilon}\left(X_{t, x}^{u}(\theta)\right)-\phi_{\mathscr{K}}^{\varepsilon}(x)\right|\right. \\
& \left.\quad \bigvee \max _{\theta \in[s, T]}\left|\phi_{\mathscr{K}}^{\varepsilon}\left(X_{s, X_{t, x}^{u}(s)}^{u}(\theta)\right)-\phi_{\mathscr{K}}^{\varepsilon}\left(X_{s, x}^{u}(\theta)\right)\right|\right] .
\end{aligned}
$$

It can be easily verified that $\phi_{\mathscr{C}}^{\varepsilon}$ and $\phi_{\mathscr{K}}^{\varepsilon}$ are Lipschitz continuous functions with Lipschitz constant $1 / \varepsilon$. Then by (8) and (9) we get

$$
\left|\vartheta^{\varepsilon}(t, x)-\vartheta^{\varepsilon}(t, y)\right| \leq \frac{1}{\varepsilon} \sup _{u \in \mathscr{U}} \mathbb{E}\left[\max _{\theta \in[t, T]}\left|X_{t, x}^{u}(\theta)-X_{t, y}^{u}(\theta)\right|\right]
$$

and

$$
\left|\vartheta^{\varepsilon}(t, x)-\vartheta^{\varepsilon}(s, x)\right| \leq \frac{1}{\varepsilon} \sup _{u \in \mathscr{U}} \mathbb{E}\left[\max _{\theta \in[t, s]}\left|X_{t, x}^{u}(\theta)-x\right| \bigvee \max _{\theta \in[s, T]}\left|X_{s, X_{t, x}^{u}(s)}^{u}(\theta)-x\right|\right]
$$

Under assumption (H1), there exists some constant $C>0$ such that for any $0 \leq t \leq$ $s \leq T, x, y \in \mathbb{R}^{d}$ the unique strong solution to equation (4) satisfies

$$
\begin{aligned}
& \mathbb{E}\left[\sup _{\theta \in[t, s]}\left|X_{t, x}^{u}(\theta)-X_{t, y}^{u}(\theta)\right|^{2}\right] \leq C|x-y|^{2}, \\
& \mathbb{E}\left[\sup _{\theta \in[t, s]}\left|X_{t, x}^{u}(\theta)-X_{s, x}^{u}(\theta)\right|^{2}\right] \leq C\left(1+|x|^{2}\right)|t-s|
\end{aligned}
$$

(see for instance [27, Theorem 6.3]).

Hence, the result follows just taking $L_{\varepsilon}:=C / \varepsilon$. 
Remark 2. It has been proved in [6, Theorem 3.1] that, if $\mathscr{K}=\mathbb{R}^{d}$ and $\mathscr{C}$ is a non empty, convex set with a $C^{1}$ regular boundary, under the uniform ellipticity condition, for some $\alpha>0, \forall(t, x, u) \in(0, T) \times \mathbb{R}^{d} \times U$,

$$
\sigma(t, x, u) \sigma(t, x, u)^{T} \geq \alpha 1_{d} .
$$

where $1_{d}$ is the identity matrix, the following holds:

$$
\left|\vartheta(t, x)-\vartheta^{\varepsilon}(t, x)\right| \leq C \frac{1+|x|^{2}+|\log \varepsilon|}{(T-t)^{d}} \varepsilon
$$

for some constant $C$ depending only on $\alpha$ and the constants in assumption (H1). We conjecture that analogous estimates can be obtained in the general case $\mathscr{K} \neq \mathbb{R}^{d}$, but a rigorous proof of this fact is still material of ongoing research.

\section{Dynamic programming and Hamilton-Jacobi-Bellman equation}

Aim of this section is to characterize the function $\vartheta^{\varepsilon}$ as a (viscosity) solution to a suitable HJB equation. For doing this, we closely follow the dynamic programming arguments recently developed in $[10,19]$ for optimal control problems with a cost depending on a running maximum. Therefore, in order to directly use those results in our framework, we will rewrite the optimal control problem (7) by means of the cost functional

$$
J(t, x, u):=\mathbb{E}\left[-\phi_{\mathscr{C}}^{\varepsilon}\left(X_{t, x}^{u}(T)\right) \bigvee \max _{\theta \in[t, T]}-\phi_{\mathscr{K}}^{\varepsilon}\left(X_{t, x}^{u}(\theta)\right)\right]
$$

such that the following holds

$$
\vartheta^{\varepsilon}(t, x)=-\inf _{u \in \mathscr{U}} J(t, x, u)
$$

The presence of the maximum operator inside the expectation, makes the cost in (14) non-Markovian preventing the direct use of the Dynamic Programming Principle (DPP), which is the first fundamental result towards the HJB characterisation. A classical strategy to overcome this difficulty consists in adding an auxiliary variable $y$ that, roughly speaking, gets rid of the non-Markovian component of the cost. This has been originally used in [8] where an approximation technique of the $L^{\infty}$-norm is used, whereas in $[10,19]$ the HJB equation is derived without making use of any approximation.

Let us introduce the following value function: 
Title Suppressed Due to Excessive Length

$$
w^{\varepsilon}(t, x, y):=\inf _{u \in \mathscr{U}} \mathbb{E}\left[-\phi_{\mathscr{C}}^{\varepsilon}\left(X_{t, x}^{u}(T)\right) \bigvee \max _{\theta \in[t, T]}-\phi_{\mathscr{K}}^{\varepsilon}\left(X_{t, x}^{u}(\theta)\right) \bigvee y\right] .
$$

Defining the process

$$
Y_{t, x, y}^{u}(.):=\max _{s \in[t, .]}-\phi_{\mathscr{K}}^{\varepsilon}\left(X_{t, x}^{u}(s)\right) \bigvee y,
$$

the value function (15) can also be written as

$$
w^{\varepsilon}(t, x, y)=\inf _{u \in \mathscr{U}} \mathbb{E}\left[-\phi_{\mathscr{C}}^{\mathcal{\varepsilon}}\left(X_{t, x}^{u}(T)\right) \bigvee Y_{t, x, y}^{u}(T)\right] .
$$

Observe that the following property holds:

$$
\vartheta^{\varepsilon}(t, x)=-w^{\varepsilon}(t, x,-1),
$$

so from now on only the optimal control problem (15) will be taken into account, since the corresponding value of the function $\vartheta^{\varepsilon}$ can be derived by the previous equality. The following property is satisfied:

Proposition 3. Let assumption (H1) be satisfied. Then, there exists a constant $C>0$ such that for any $\varepsilon>0, t, s \in[0, T],(x, y),\left(x^{\prime}, y^{\prime}\right) \in \mathbb{R}^{d+1}$ one has

$$
\left|w^{\varepsilon}(t, x, y)-w^{\varepsilon}\left(s, x^{\prime}, y^{\prime}\right)\right| \leq \frac{C}{\varepsilon}\left(\left|x-x^{\prime}\right|+\left|y-y^{\prime}\right|+|t-s|^{\frac{1}{2}}\left(1+|x| \vee\left|x^{\prime}\right|\right)\right) .
$$

Moreover, for any family of stopping times $\left\{\tau^{u}, u \in \mathscr{U}\right\}$ with values in $[t, T]$ one has

$$
w^{\varepsilon}(t, x, y)=\inf _{u \in \mathscr{U}} \mathbb{E}\left[w^{\varepsilon}\left(\tau^{u}, X_{t, x}^{u}\left(\tau^{u}\right), Y_{t, x, y}^{u}\left(\tau^{u}\right)\right)\right]
$$

for any $(t, x, y) \in[0, T] \times \mathbb{R}^{d+1}$.

Proof. The regularity of $w^{\varepsilon}$ with respect to $t$ and $x$ can be proved as in Proposition 2 , while the Lipschitzianity with respect to $y$ is trivial.

Thanks to the regularity of $w^{\varepsilon}$, the DPP (17) follows by arguments similar to [12] observing that for the couple of variables $\left(X_{t, x}^{u}(\cdot), Y_{t, x, y}^{u}(\cdot)\right)$ the following property holds:

$$
\left(\begin{array}{l}
X_{t, x}^{u}(s) \\
Y_{t, x, y}^{u}(s)
\end{array}\right)=\left(\begin{array}{l}
X_{\theta, X_{t, x}^{u}(\theta)}^{u}(s) \\
Y_{\theta, X_{t, x}^{u}(\theta), Y_{t, x, y}^{u}(\theta)}^{u}(s)
\end{array}\right) \quad \text { a.s. }
$$

for any $t \leq \theta \leq s \leq T$ ( with $\theta$ possibly a stopping time). We remand to [10] for a sketch of the proof showing how the arguments in [12] adapt to our case. 


\subsection{HJB equation}

Proposition 3 is the main tool for proving the next result that characterizes $w^{\varepsilon}$ as the unique solution, in the viscosity sense, of a suitable HJB equation. In the sequel we will restrict our domain to

$$
D:=\left\{(x, y) \in \mathbb{R}^{d+1}:-\phi_{\mathscr{K}}^{\varepsilon}(x)<y<0\right\} .
$$

Indeed, the knowledge of $w^{\varepsilon}$ in $\bar{D}$ is sufficient to characterize it everywhere thanks to the following relation:

$$
\begin{array}{ll}
w^{\varepsilon}(t, x, y)=y & \text { for any } y \geq 0 \\
w^{\varepsilon}(t, x, y)=w^{\varepsilon}\left(x,-\phi_{\mathscr{K}}^{\varepsilon}(x)\right) & \text { for any } y \leq-\phi_{\mathscr{K}}^{\varepsilon}(x) .
\end{array}
$$

Based on this observation, it is sufficient to characterise $w^{\varepsilon}$ in the domain $\bar{D}$. Letting

$$
\Gamma_{1}:=\{(x, y) \in \bar{D}: y=0\} ; \quad \Gamma_{2}:=\left\{(x, y) \in \bar{D}: y=-\phi_{\mathscr{K}}^{\varepsilon}(x)\right\},
$$

we are going to prove that $w^{\varepsilon}$ is the unique solution (in the weak sense specified in Definition 1 below) of the following HJB equation with mixed derivative-Dirichlet boundary conditions:

$$
\left\{\begin{array}{lr}
-\partial_{t} w+H\left(t, x, D_{x} w, D_{x}^{2} w\right)=0 & {[0, T) \times D} \\
w=0 & {[0, T) \times \Gamma_{1}} \\
-\partial_{y} w=0 & {[0, T) \times \Gamma_{2}} \\
w(T, x, y)=w_{0}(x, y) & \bar{D}
\end{array}\right.
$$

with

$$
H(t, x, p, Q):=\sup _{u \in U}\left\{-b(t, x, u) p-\frac{1}{2} \operatorname{Tr}\left[\sigma \sigma^{T}\right](t, x, u) Q\right\}
$$

and

$$
w_{0}(x, y):=-\phi_{\mathscr{C}}^{\varepsilon}(x) \bigvee-\phi_{\mathscr{K}}^{\varepsilon}(x) \bigvee y .
$$

We point out that the derivative boundary condition $-\partial_{y} w=0$ on $\Gamma_{2}$ is typically obtained in presence of a running maximum cost, see $[8,10]$, while the Dirichlet condition $w^{\varepsilon}=0$ on $\Gamma_{1}$ is obtained by the very definition of $w^{\varepsilon}$. Observe also that the constant Dirichlet condition on $\Gamma_{1}$ is compatible with the homogeneus derivative condition on $\Gamma_{2}$. This prevents possible problems related with mixed boundary conditions at the junctions where different components of the boundary cross.

The fully nonlinearity and degeneracy of the equation requires to consider solutions in the viscosity sense (see [14] for an overview on the subject). This notion of solution requires also to specify in which sense boundary conditions are satisfied. In paricular, we ask the Dirichlet conditions on $\Gamma_{1}$ to be satisfied in the strong sense, whereas the derivative conditions on $\Gamma_{2}$ are considered in the (weak) viscosity sense.

Definition 1. A USC function $\underline{w}$ (resp. LSC function $\bar{w}$ ) on $[0, T] \times \bar{D}$ is a viscosity sub-solution (resp. super-solution) of (20), if for every function $\varphi \in C^{1,2}([0, T] \times \bar{D})$, 
at each maximum (resp. minimum) point $(t, x, y)$ of $\underline{w}-\varphi(\operatorname{resp} . \bar{w}-\varphi)$ the following inequality holds

$$
\left\{\begin{array}{lc}
-\partial_{t} \varphi+H\left(t, x, D_{x} \varphi, D_{x}^{2} \varphi\right) \leq 0 & {[0, T) \times D} \\
\varphi \leq 0 & {[0, T) \times \Gamma_{1}} \\
\min \left(-\partial_{y} \varphi,-\partial_{t} \varphi+H\left(t, x, D_{x} \varphi, D_{x}^{2} \varphi\right)\right) \leq 0[0, T) \times \Gamma_{2} \\
\varphi(T, x, y) \leq w_{0}(x, y) & \bar{D}
\end{array}\right.
$$

(resp.

$$
\left\{\begin{array}{lc}
-\partial_{t} \varphi+H\left(t, x, D_{x} \varphi, D_{x}^{2} \varphi\right) \geq 0 & {[0, T) \times D} \\
\varphi \geq 0 & {[0, T) \times \Gamma_{1}} \\
\max \left(-\partial_{y} \varphi,-\partial_{t} \varphi+H\left(t, x, D_{x} \varphi, D_{x}^{2} \varphi\right)\right) \geq 0 & {[0, T) \times \Gamma_{2}} \\
\varphi(T, x, y) \geq w_{0}(x, y) & \bar{D} .
\end{array}\right.
$$

A continuous function $w$ on $[0, T] \times \bar{D}$ is a viscosity solution of (20) if it is both a sub- and super-solution.

Theorem 1. Let assumption (H1) be satisfied. Then, $w^{\varepsilon}$ is the unique bounded and continuous viscosity solution of the HJB equation (20).

Proof. The Dirichlet and terminal conditions are ensured by the very definition of $w^{\varepsilon}$ and its continuity. In particular, the continuity allows the conditions to be considered in the strong sense. The proof of sub- and supersolution properties in $[0, T) \times\left(D \cup \Gamma_{2}\right)$ follows quite straightforward by the the arguments in [10, Theorem 3.2] and [21, Theorem 4.1].

Uniqueness of the solution relies on comparison results for sub and super solution. The proof can be found in [19, Appendix A]. We point out that the fact of considering Dirichlet conditions in a strong sense is an important requirement for the proof of the comparison principle.

\section{Numerical Approximation}

In this section we discuss an approximation scheme for the unique continuous viscosity solution $w^{\varepsilon}$ to the equation

$$
\begin{aligned}
\partial_{t} w+H\left(t, x, D_{x} w, D_{x}^{2} w\right) & =0 & & -\phi_{\mathscr{K}}^{\varepsilon}(x)<y<0, t \in(0, T] \\
w & =0 & & y=0, t \in(0, T] \\
-\partial_{y} w & =0 & & y=-\phi_{\mathscr{K}}^{\varepsilon}(x), t \in(0, T]
\end{aligned}
$$

with initial data

$$
w(0, x, y)=w_{0}^{\varepsilon}(x, y) \quad-\phi_{\mathscr{K}}^{\varepsilon}(x) \leq y \leq 0
$$

(the convenient change of variable $t \rightarrow T-t$ has been here applied). 
In [10, Section 4.1] a general convergence result for numerical schemes approximating HJB equations under oblique derivative boundary conditions as (22c) is provided. Those arguments can be easily modified in order to prove convergence also in presence of the additional Dirichlet boundary condition (22b) (see also [21]). Following the ideas introduced in [10], we present here a semi-Lagrangian (SL) scheme for the approximation of (22). The same scheme will be used in the numerical experiments in Section 5.

Let $N \geq 1$ be an integer (number of time steps), and let

$$
h:=\frac{T}{N} \quad \text { and } \quad t_{n}:=n h
$$

for $n=0, \ldots, N$. Let $\Delta x=\left(\Delta x_{1}, \ldots, \Delta x_{d}\right) \in\left(\mathbb{R}_{+}^{*}\right)^{d}$ and $\Delta y>0$, and let $\mathscr{G}_{\rho}$ (where $\rho \equiv(\Delta x, \Delta y))$ be the space grid

$$
\mathscr{G}_{\rho}:=\left\{\left(x_{i}, y_{j}\right)=(i \Delta x, j \Delta y), \text { for }(i, j) \in \mathbb{Z}^{d} \times \mathbb{Z}\right\} .
$$

The grid is considered uniform for simplicity of presentation. We also assume that the discretization in the $y$ coordinate is aligned with the boundary of the domain, this allows us to get the Dirichlet condition exactly.

We look for a fully discrete scheme for the viscosity solution of (22) on the timespace grid $\left\{t_{0}, \ldots, t_{N}\right\} \times\left(\mathscr{G}_{\rho} \cap \bar{D}\right)$. Following the ideas in $[10,21]$ the numerical scheme is defined starting from a standard scheme for (22a), which is then mixed with a step of "projection" on $\Gamma_{2}$ and the use of the Dirichlet condition on $\Gamma_{1}$. The approximation of equation (22a) we consider is the SL scheme proposed by Camilli and Falcone [13] and also used in [15]. We recall that first schemes of this type have been introduced by Menaldi in [23].

Let $\sigma^{u}=\sigma(\cdot, \cdot, u)$ and $b^{u}=b(\cdot, \cdot, u)$, and let $\left(\sigma_{k}^{u}\right)_{k=1, \ldots, p}$ denote the column vectors of $\sigma^{u}$. We consider the following operator $\mathscr{T}$ :

$$
\mathscr{T}(\varphi)(t, x, y):=\min _{u \in U} \frac{1}{2 p}\left(\sum_{k=1, \ldots, 2 p}[\varphi(t, . ., y)]\left(x+h b^{u}(t, x)+\sqrt{h} \bar{\sigma}_{k}^{u}(t, x)\right)\right)
$$

with the following vector definition in $\mathbb{R}^{d}$ :

$$
\bar{\sigma}_{2 k-j}^{u}:=\sqrt{p}(-1)^{j} \sigma_{k}^{u}
$$

for $k=1, \ldots, p$ and $j \in\{0,1\}$. Now $[\cdot] \equiv[\cdot]_{x}$ stands for a monotone, $P_{1}$ interpolation operator on the $x$-grid $\left(x_{i}\right)$, satisfying in particular:

$$
\left\{\begin{array}{l}
(i)[\varphi]\left(x_{i}\right)=\varphi\left(x_{i}\right), \text { for any } i \in \mathbb{Z}^{d}, \\
\text { (ii) }|[\varphi](x)-\varphi(x)| \leq C|\Delta x|^{2}\left\|D_{x}^{2} \varphi\right\|_{\infty} \text { for any } \varphi \in C^{2}\left(\mathbb{R}^{d}, \mathbb{R}\right), \\
\text { (iii) for any functions } \varphi, \psi: \mathbb{R}^{d} \rightarrow \mathbb{R}, \varphi \leq \psi \Rightarrow[\varphi] \leq[\psi] .
\end{array}\right.
$$

We point out that (23), if considered without interpolation, is a discretization in time of the Dynamic Programming Principle. In particular, such an approximation 
uses an Euler-Maruyama scheme (see [20] for instance) coupled with a finite state discretization of the Gaussian discribution to approximate the dynamics $X_{t, x}^{u}(\cdot)$.

The numerical scheme is defined as follows:

Algorithm. Initialization step, for $n=0$, for all $i, j$ :

$$
W_{i, j}^{0}=w_{0}^{\varepsilon}\left(x_{i}, y_{j}\right)
$$

Then, for $n=0, \ldots, N-1$ :

Step 1 Compute $W_{i, j}^{n+1}=\mathscr{T}(W)\left(t_{n}, x_{i}, y_{j}\right)$, for all $\left(x_{i}, y_{j}\right) \in \mathscr{G}_{\rho} \cap \bar{D}$;

Step 2 Assign $W_{i, j}^{n+1}=W_{i, j_{i}}^{n+1}$, for all $\left(x_{i}, y_{j}\right): y_{j} \leq-\phi_{\mathscr{K}}^{\varepsilon}\left(x_{i}\right)$;

$$
W_{i, j}^{n+1}=y_{j} \text {, for all }\left(x_{i}, y_{j}\right): y_{j} \geq 0
$$

where for every $x \in \mathbb{R}^{d}, j_{x} \in \mathbb{Z}$ is defined by

$$
j_{x}:=\min \left\{j \in \mathbb{Z}: j \Delta y \geq-\phi_{\mathscr{K}}^{\varepsilon}(x)\right\}
$$

and we also used the following short notation

$$
W_{i, j}^{n}=W\left(t_{n}, x_{i}, y_{j}\right) .
$$

Hereafter we will denote by $W=\left(W_{i j}^{n}\right)_{(i, j) \in \mathbb{Z}^{d+1}}^{n=1 \ldots N}$ the solution of the numerical scheme defined by the algorithm above on $\left\{t_{0}, \ldots, t_{N}\right\} \times \mathscr{G}_{\rho}$. We point out that the necessity of defining $W$ also at mesh points outside $\bar{D}$ comes from the fact that the SL scheme involves values outside the domain. However, this is not a issue in virtue of (18) (see Step 2 above).

We also denote by $W^{\rho, h}$ the continuous extension of $W$ to $[0, T] \times \mathbb{R}^{d} \times \mathbb{R}$ obtained by linear interpolation.

Remark 3. The numerical solution $W^{\rho, h}$ is Lipschitz continuous in $y$ with Lipschitz constant independent of $\rho$ and $h$. This can be derived by the very definition of the operator $\mathscr{T}$ in (23). Indeed, given $W^{n} L$-Lipschitz continuous in $y$ one can observe that

$$
\begin{aligned}
\left|W_{i, j}^{n+1}-W_{i, j^{\prime}}^{n+1}\right| & =\left|\mathscr{T}(W)\left(t_{n}, x_{i}, y_{j} \vee y_{j_{x_{i}}}\right)-\mathscr{T}(W)\left(t_{n}, x_{i}, y_{j^{\prime}} \vee y_{j_{x_{i}}}\right)\right| \\
& \leq L\left|\left(y_{j} \vee y_{j_{x_{i}}}\right)-\left(y_{j^{\prime}} \vee y_{j_{x_{i}}}\right)\right| \leq L\left|y_{j}-y_{j^{\prime}}\right|
\end{aligned}
$$

Hence, being $W^{0}$ Lipschitz continuous, the same property holds for $W^{n}$ for all $n=$ $1 \ldots N$. Then, since the linear interpolation (used to pass from $W$ to $W^{\rho, h}$ ) preserve Lipschitz constants, we can obtain the desired property.

Remark 4. The core of the scheme in Step 1 can be written as

$$
S\left(t, x, y, W_{i, j}^{n+1}, W\right):=W_{i, j}^{n+1}-\mathscr{T}(W)\left(t_{n}, x_{i}, y_{j}\right)=0 .
$$


It is immediate to verify that $S$ is monotone in the sense of Barles and Souganidis [7], i.e. for every $h, \rho>0, r \in \mathbb{R}$, for all function $\phi, \psi$ such that $\phi \geq \psi$, inequality

$$
S(t, x, y, r, \phi) \leq S(t, x, y, r, \psi)
$$

holds.

The choice of $\bar{\sigma}_{k}^{u}$ in (24) leads to the following consistency estimate, for any $\varphi \in C^{2,4}\left((0, T) \times \mathbb{R}^{d} \times \mathbb{R}\right):$

$$
\begin{aligned}
& \left|\frac{1}{h} S(t, x, y, \varphi(t, x, y), \phi)-\left(\partial_{t} \varphi+H\left(t, x, D_{x} \varphi, D_{x}^{2} \varphi\right)\right)\right| \\
& \leq C_{1}\left(\left|b^{u}(t, x)\right|^{2}\left\|D_{x}^{2} \varphi\right\|_{\infty}+\left|b^{u}(t, x)\left\|\left.\sigma^{u}(t, x)\right|^{2}\right\| D_{x}^{3} \varphi\left\|_{\infty}+\left|\sigma^{u}(t, x)\right|^{4}\right\| D_{x}^{4} \varphi \|_{\infty}\right.\right. \\
& \left.\left\|\partial_{t t}^{2} \varphi\right\|_{\infty}\right) h+C_{2}\left\|D_{x}^{2} \varphi\right\|_{\infty} \frac{|\Delta x|^{2}}{h} .
\end{aligned}
$$

These are classical properties of SL schemes, see [15] for instance. In particular, the error term in $|\Delta x|^{2} / h$ comes the interpolation error estimate (ii) (observe that we do not need to interpolate with respect to $y$ ) and the term in $h$ from classical Taylor expansions. Then, in order to ensure consistency of the scheme, $\Delta x$ and $h$ have to be chosen so that $|\Delta x|^{2} / h \rightarrow 0$ as $\Delta x, h \rightarrow 0$. This usually leads to the choice $\Delta x \sim h$ in numerical simulations.

Moreover, it is easy to verify that the scheme admits a bounded solution in $\left\{t_{0}, \ldots, t_{N}\right\} \times\left(\mathscr{G}_{\rho} \cap \bar{D}\right)$, so that the scheme is also stable.

We recall that monotonicity, consistency and stability are the fundamental properties necessary for proving convergence of numerical schemes in the framework of viscosity solutions, see [7].

Theorem 2. Let assumption (H1) be satisfied. Let $W^{\rho, h}$ be the solution of the scheme defined by Algorithm 1, where $\mathscr{T}$ is the SL scheme (23)-(24). Then, if

$$
\frac{|\Delta x|^{2}}{h} \rightarrow 0 \quad \text { as } \quad \Delta x, h \rightarrow 0
$$

$W^{\rho, h}$ converges to $w^{\varepsilon}$ in $[0, T] \times \bar{D}$ as $\rho, h \rightarrow 0$.

Proof. The proof follows the strategy in [7] and [10]. Let us define for $(t, x, y) \in$ $[0, T] \times \bar{D}$

$$
\begin{aligned}
& \bar{W}(t, x, y):=\limsup _{\substack{[0, T] \times \bar{D} \ni(s, \xi, \gamma) \rightarrow(t, x, y) \\
\rho, h \rightarrow 0}} W^{\rho, h}(s, \xi, \gamma), \\
& \underline{W}(t, x, y):=\liminf _{\substack{0, T] \times \bar{D} \ni(s, \xi, \gamma) \rightarrow(t, x, y) \\
\rho, h \rightarrow 0}} W^{\rho, h}(s, \xi, \gamma) .
\end{aligned}
$$


One clearly has $\underline{W}(t, x, y) \leq \bar{W}(t, x, y)$ for any $(t, x, y) \in[0, T] \times \bar{D}$. Convergence follows by the comparison principle once shown that $\bar{W}$ and $\underline{W}$ are respectively a sub- and supersolution of the HJB equation in the sense of Definition 1.

We sketch the proof of the subsolution property, the supersolution part can be proved in a similar way. Given a smooth test function $\varphi$, let $(\bar{t}, \bar{x}, \bar{y})$ be a maximum point for $(\underline{W}-\varphi)$, with $(\underline{W}-\varphi)(\bar{t}, \bar{x}, \bar{y})=0$, and let $\left(\rho_{k}, h_{k}, t_{k}, x_{k}, y_{k}\right)$ be such that $\left(t_{k}, x_{k}, y_{k}\right) \in[0, T] \times \bar{D}, \rho_{k}, h_{k} \rightarrow 0,\left(t_{k}, x_{k}, y_{k}\right) \rightarrow(\bar{t}, \bar{x}, \bar{y}), W^{\rho_{k}, h_{k}}\left(t_{k}, x_{k}, y_{k}\right) \rightarrow$ $\underline{W}(\bar{t}, \bar{x}, \bar{y})$ and

$$
\left(W^{\rho_{k}, h_{k}}-\varphi\right)\left(t_{k}, x_{k}, y_{k}\right)=\max \left(W^{\rho_{k}, h_{k}}-\varphi\right)=\delta_{k} \rightarrow 0
$$

(the existence of such a sequence follows by classical arguments in viscosity theory).

If $(\bar{x}, \bar{y}) \in D$ the result follows as in [7] using the prperties of monotonicity and consistency of the scheme in a sufficiently small neighborhood of $(\bar{x}, \bar{y})$ still contained in $D$.

If $(\bar{x}, \bar{y}) \in \Gamma_{2}$ one can work under the condition $-\partial_{y} \varphi(\bar{t}, \bar{x}, \bar{y})>0$, otherwise the subsolution property is automatically satisfied. In this case the result follows observing that, by the very definition of the scheme (see Step 2 of the algorithm) and its monotonicity, one can derive

$$
\varphi\left(t_{k}, x_{k}, y_{k}\right)+\delta_{k} \leq \mathscr{T}(\varphi)\left(t_{k}, x_{k}, y_{k} \vee y_{j_{x_{k}}}\right) \leq \mathscr{T}(\varphi)\left(t_{k}, x_{k}, y_{k}\right)
$$

so that the subsolution property follows again by the consistency of the scheme.

It remains to prove that $\underline{W}$ satisfies the Dirichlet condition pointwise on $\Gamma_{1}$. For this purpose it is worth to observe that $W^{\rho, h}$ is Lipschitz continuous in $y$ (see Remark 3), i.e. there exists some constant $L>0$ (independent of $\rho, h, t, x$ ) such that

$$
\left|W^{\rho, h}(t, x, y)-W^{\rho, h}\left(t, x, y^{\prime}\right)\right| \leq L\left|y-y^{\prime}\right|
$$

for any $t \in[0, T], x \in \mathbb{R}^{d}, y, y^{\prime} \in \mathbb{R}$. Therefore one has

$$
\left|W^{\rho, h}(s, \xi, \gamma)-(-1)\right|=\left|W^{\rho, h}(s, \xi, \gamma)-W^{\rho, h}(s, \xi, 0)\right| \leq L|\gamma|
$$

so that on $\Gamma_{1}$ (i.e for $\bar{y}=0$ )

$$
\liminf _{[0, T] \times \bar{D} \ni(s, \xi, \gamma) \rightarrow(\bar{t}, \bar{x}, \bar{y})} W^{\rho, h \rightarrow 0}(s, \xi, \gamma)=-1 .
$$

\section{Numerical tests}

In this section we present some numerical tests for probabilistic reachability problems in presence of state constraints. To solve the HJB equation (22), we use the fully discrete SL scheme introduced in Section 4 implemented on the ROC-HJ 
solver available at the link https: / / uma .ensta-paristech.fr/soft/ROC-HJ/. The minimum in (23) is performed on a subset of control values $\left\{u_{1}, \ldots, u_{N_{u}}\right\}$ that represents a discretization of $U$ with a mesh size $\Delta u$. In all the simulations the regularization parameter will be chosen to be $\varepsilon=1 . E-08$.

\subsection{Example 1}

We consider the following controlled stochastic system:

$$
\mathrm{d} X(s)=\left(\left(\begin{array}{cc}
-1 & -4 \\
4 & -1
\end{array}\right) X(s)+u(s)\right) \mathrm{d} s+\left(\begin{array}{cc}
0.7 & 0 \\
0 & 0.7
\end{array}\right)\left(\begin{array}{l}
\mathrm{d} B_{1}(s) \\
\mathrm{d} B_{2}(s)
\end{array}\right)
$$

where $u(s)=\left(\begin{array}{l}u_{1}(s) \\ u_{2}(s)\end{array}\right), u_{i}(s) \in[-0.1,0.1]$, for $i=1,2$ and $B_{1}, B_{2}$ are two independent Brownian motions.

The linear system (27) has been used in [6] to validate the HJB approach in the characterization of an approximated probabilistic reachable set without state constraints and in [5] to illustrate an appoximation of the probability of reaching a target by using enclosing hulls of probability density functions.

We set $T=1.75$ and define the target $\mathscr{C}:=(0.5,1.5) \times(-0.5,0.5)$ (green square in Figure 2). The constraint is given by the presence of an obstacle, represented in Figure 2 (right) by the red rectangle, i.e. $\mathscr{K}:=\mathbb{R}^{2} \backslash([-6,-2] \times[2,4])$. We compute the set $\Omega_{t}^{\rho}$ (blue region) for $t=0.75$ and $\rho=0.4$ in presence (Figure 2, right) or not (Figure 2, left) of the obstacle. To approximate the auxiliary function $w^{\mathcal{E}}$ solution to (22), the numerical simulation is performed on a computational domain $[-8,8]^{2} \times[-1,0]$. The corresponding values of $\vartheta^{\varepsilon}$ are then obtained using relation (16).

Figure 3 (top) shows the set $\Omega_{t}^{\rho}$ for $\rho=0.4$ at different time $t \in\{0.25 ; 0\}$ in presence of the obstacle. Then, in Figure 3 (bottom) we simulate different optimal paths starting from a given point of the backward reachable set using the algorithm described below.

Algorithm (trajectory reconstruction) Initialization: Set $X_{0}=\bar{x}$.

For $k=0$ to $N-1$ :

Step 1 Compute optimal control at $t=t_{k}$ :

$$
u^{k}=\arg \min _{u \in\left\{u_{1}, \ldots, u_{N_{u}}\right\}} \mathbb{E}\left[W\left(t_{k+1}, X_{k+1}^{u},-1\right)\right]
$$

where for $u_{i} \in\left\{u_{1}, \ldots, u_{N_{u}}\right\}$

$$
X_{k+1}:=X_{k}+b\left(t_{k}, X_{k}, u_{i}\right) h+\sigma\left(t_{k}, X_{k}, u_{i}\right) \sqrt{h} \xi,
$$



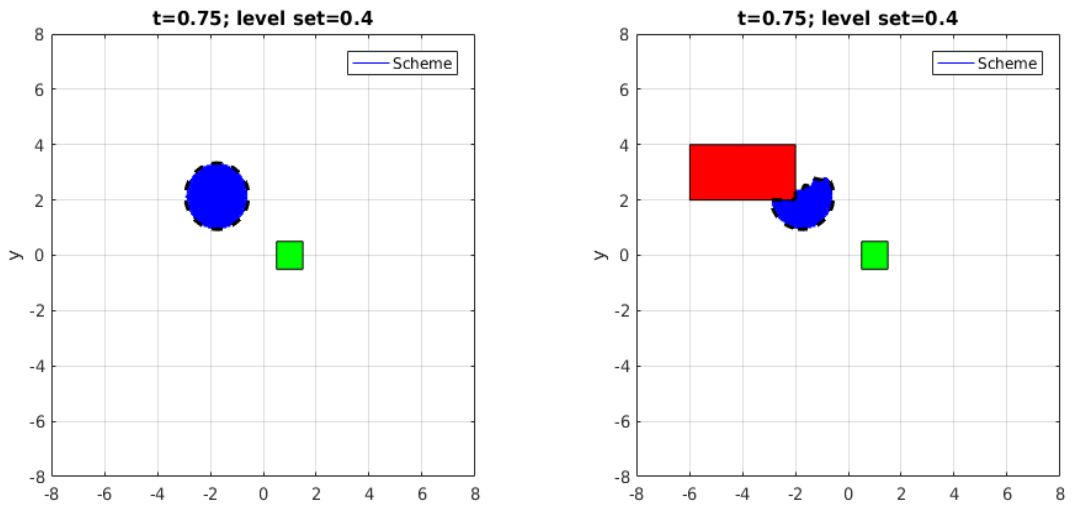

Fig. 2 (Example 1) Backward reachable sets at $t=0.75$ for a time hor̀zon $T=1.75$ and $\rho=0.4$ without (left) and with (right) obstacle. The target set, the obstacle and the backward reachable set $\Omega_{0.75}^{0.4}$ are represented respectively by the green square, the red rectangle and the blue region.

here $\xi:=\left(\xi_{1}, \xi_{2}\right)$ with $\xi_{i}(i=1,2)$ random variables following a $N(0,1)$ distribution.

Step 2 Compute the point of the optimal trajectory:

$$
X_{k+1}:=X_{k}+b\left(t_{k}, X_{k}, u^{k}\right) h+\sigma\left(t_{k}, X_{k}, u^{k}\right) \sqrt{h} \xi
$$

where again $\xi_{i} \sim N(0,1)$ for $i=1,2$.

In order to validate our approach, we compare the value of the scheme in a given point with the percentage of trajectories reaching the target without hitting the obstacle. We consider the case $t=0.25$ and two different starting points $\bar{x}_{1}:=(4.0,1.0)^{T}$ and $\bar{x}_{2}:=(3.0,0.0)^{T}$. The approximation of the level-set function obtained by numerically solving the corresponding HJB equation on the grid $\Delta x_{1}=\Delta x_{2}=0.0125, \Delta y=0.1, h=0.025$ at points $\bar{x}_{1}$ and $\bar{x}_{2}$ is respectively $-W\left(t, \bar{x}_{1},-1\right) \simeq 0.459 \pm 0.004$ and $-W\left(t, \bar{x}_{2},-1\right) \simeq 0.404 \pm 0.009$.

The results of Monte Carlo simulations are reported in Table 1. One can conlcude that the approximated value of the level set function belongs in each case to the confidence interval.

\subsection{Example 2.}

We now test our method on the same example used in [10, Section 6]. Let us consider the following dynamics: 

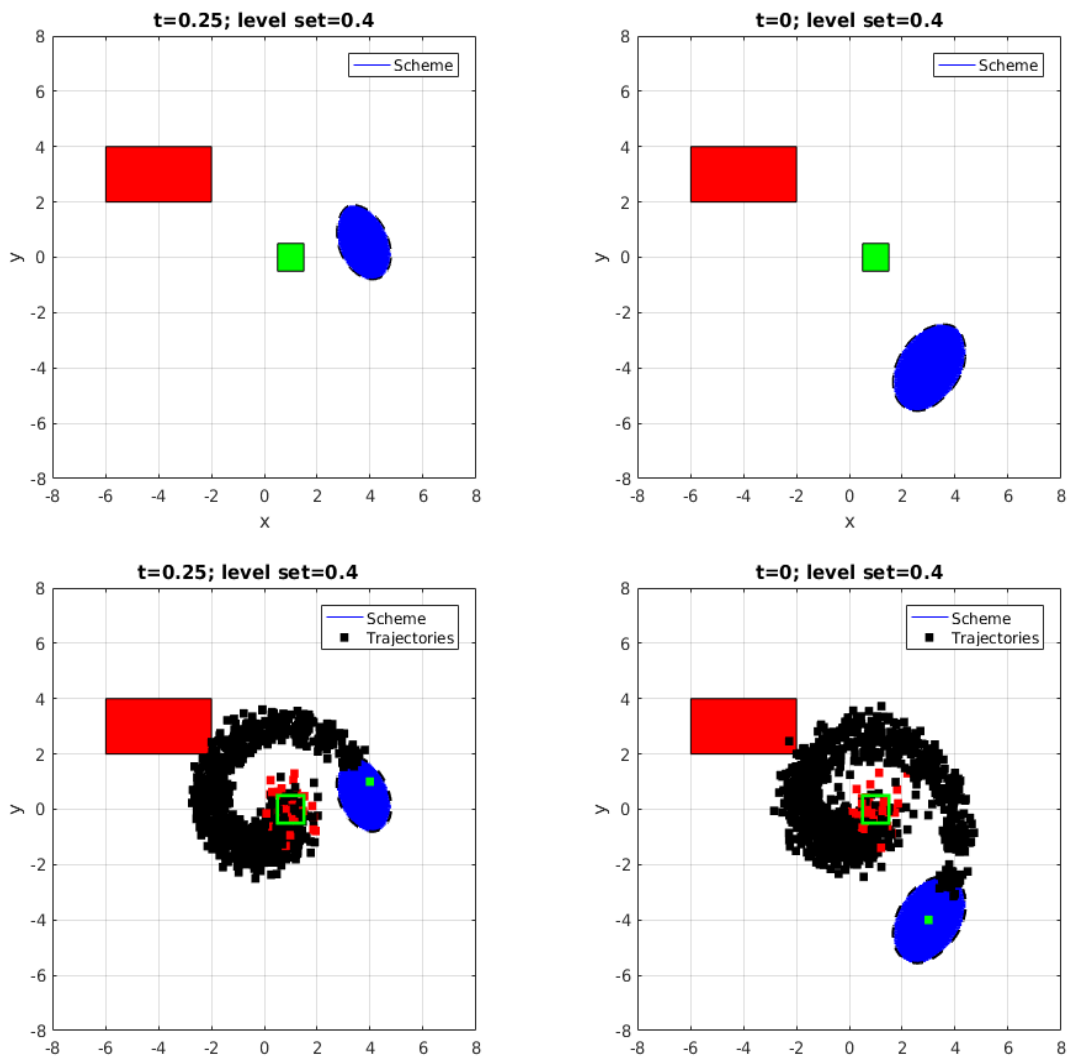

Fig. 3 (Example 1). ${ }^{\times}$Top: backward reachable set (blue region) at times $t \in\{0.25,0\}$ for a final time horizon $T=1.75$ in presence of the obstacle (red rectangle). Bottom: reconstruction of some optimal paths starting from point $\bar{x}=(4,1)$ (bottom, left) and $\bar{x}=(3,-4)$ (bottom, right).

\begin{tabular}{|r|r|c|c|c|}
\hline & $M$ & $p$ & C.I. & MC-error \\
\hline & 6000 & 0.4630 & $(0.4504,0.4756)$ & 0.0126 \\
& 12000 & 0.4624 & $(0.4535,0.4713)$ & 0.0089 \\
$\bar{x}_{1}$ & 25000 & 0.4603 & $(0.4541,0.4664)$ & 0.0062 \\
& 50000 & 0.4618 & $(0.4574,0.4661)$ & 0.0045 \\
& 100000 & 0.4628 & $(0.4597,0.4659)$ & 0.0031 \\
\hline \multirow{4}{*}{$\bar{x}_{2}$} & 6000 & 0.3915 & $(0.3593,0.4037)$ & 0.0122 \\
& 12000 & 0.3991 & $(0.3705,0.4078)$ & 0.0087 \\
& 25000 & 0.4026 & $(0.3966,0.4087)$ & 0.0061 \\
& 50000 & 0.4016 & $(0.3996,0.4081)$ & 0.0043 \\
& 100000 & 0.4015 & $(0.3985,0.4045)$ & 0.0030 \\
\hline
\end{tabular}

Table 1 (Example 1) Percentage $p$ of $M$ simulated trajectories that reach the target set without hitting the obstacle, with a corresponding confidence interval (C.I.), and a Monte Carlo error estimate (MC-error) 


$$
\mathrm{d} X(s)=u(s)\left(\begin{array}{l}
1 \\
0
\end{array}\right) \mathrm{d} s+u(s) \sigma(X(s)) \mathrm{d} B(s), s \geq t,
$$

where $B$ is a one-dimensional Brownian motion, $U=[0,1] \subset \mathbb{R}$ and the volatility $\sigma(x)$ is given by

$$
\sigma(x):=5 d_{\Theta}(x)\left(\begin{array}{l}
0 \\
1
\end{array}\right)
$$

where $d_{\Theta}$ denotes the distance function to the set

$$
\Theta:=\left\{\left(x_{1}, x_{2}\right),\left|x_{2}\right| \geq 0.3\right\} .
$$

The target set is $\mathscr{C}=(0,0.4) \times(-0.5,0.5)$ (green rectangle in Figure 4) and the state constraint is $\mathscr{K}=\mathbb{R}^{2} \backslash([-0.4,0.2] \times[-0.1,0.1])$ (i.e. the entire space except the red square obstacle in Figure 4). We fix $T=0.5$ and consider the computational domain $[-1,1]^{2} \times[-1,0]$.

The strong degeneracy of the diffusion term in this example allowed in [10] to obtain the "almost sure" backward reachable set, corresponding here to the case $\rho=1$. Figure 4 shows the approximation of $\Omega_{t}^{\rho}$ for $t=0$ and different levels $\rho \in$ $[0,1,0.9]$. The black region corresponds to the exact backward reachable set for $\rho=1$. Indeed, due to the simple dynamics considered it is possible for this example to infer the exact set $\Omega_{0}^{1}$, i.e. the set of points from where the target is reached and the constraint satisfied with probability one, see [10] for a further discussion. One can observe that as $\rho$ approaches the value 1, we recover the results obtained in [10]. A loss of precision appears at corners. This was already noticed in [10] and it is due to the smoothing effects of the diffusion term (see [10, Figure 2, Section 6]) which can be reduced with the refinement of the mesh.

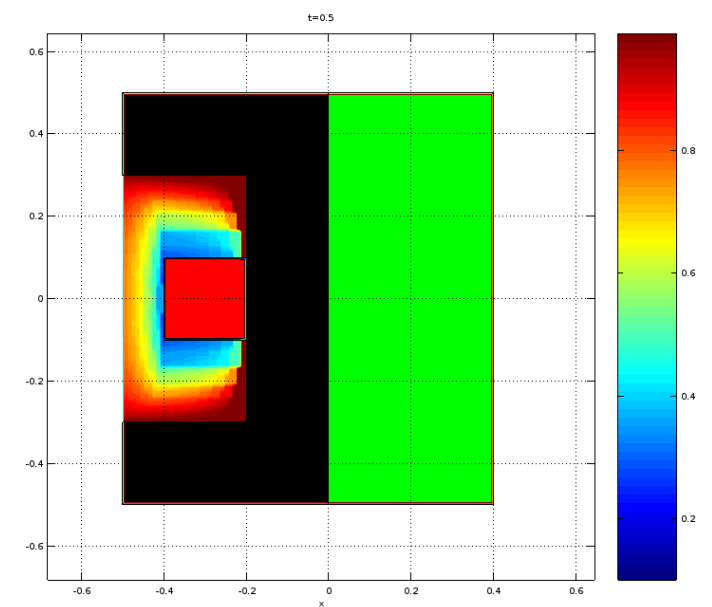

Fig. 4 (Example 2) approximation of $\Omega_{t}^{\rho}$ at $t=0$ for different levels $\rho \in[0,1,0.9]$ computed with $\Delta x_{1}=\Delta x_{2}=0.005, \Delta y=0.1, h=0.01$ (same mesh parameters used in [10]). 


\section{Conclusions}

In this paper we have used the HJB theory for characterising the probabilistic backward reachable set for a system of controlled diffusions in presence of state constraints. We have shown that such a set is a level set of the value function associated to a suitable optimal control problem. To deal with the discontinuity of the cost functional associated to this problem, arising from the use of indicator functions for representing probabilities, we have defined a regularised problem. Precise estimates of the error introduced by this regularisation are still object of ongoing research.

Following the approach in $[10,19,21]$, for the regularised problem we have obtained a characterization by a HJB equation with mixed Dirichlet-derivative boundary conditions. We have defined a fully discrete SL approximation scheme and we have proved its convergence to the unique viscosity solution of the equation. Then, we have used such a scheme in order to validate our approach on some numerical tests. We focused on the examples studied in [6] and [10], adding state constraints to the first one and variable levels of probability to the second one. More complex tests on concrete models are a promising future direction of work.

Acknowledgements The authors are sincerely grateful to Olivier Bokanowski and Hasnaa Zidani for their guidance at the early stage of this paper.

\section{References}

1. A. Abate, .S Amin, M. Prandini, J. Lygeros, and S. Sastry. Computational approaches to reachability analysis of stochastic hybrid systems. Hybrid Systems, LNCS 4416(1):4-17, 2007.

2. A. Abate, M. Prandini, J. Lygeros, and S. Sastry. Probabilistic reachability and safety for controlled discrete time stochastic hybrid systems. Automatica, 44:2724-2734, 2008.

3. M. Althoff, O. Stursberg, and M. Buss. Safety assessment of autonomous cars using verification techniques. In 2007 American Control Conference, pages 4154-4159, 2007.

4. M. Althoff, O. Stursberg, and M. Buss. Safety assessment for stochastic linear systems using enclosing hulls of probability density functions. In 2009 European Control Conference (ECC), pages 625-630, 2009.

5. M. Althoff, O. Stursberg, and M. Buss. Safety assessment for stochastic linear systems using enclosing hulls of probability density functions. In 10th European Control Conference, pagesto appear, 2009.

6. M. Assellaou, O. Bokanowski, and H. Zidani. Error estimates for second order hamiltonjacobi-bellman equations. approximation of probabilistic reachable sets. Discrete and Continuous Dynamical Systems - Serie A, 35(9):3933 - 3964, 2015.

7. G. Barles and P.E. Souganidis. Convergence of approximation schemes for fully nonlinear second order equations. Asymptotic Anal., 4:271-283, 1991.

8. E. N. Barron. The Bellman equation for control of the running max of a diffusion and applications to lookback options. Applicable Analysis, 48:205-222, 1993.

9. O. Bokanowski, N. Forcadel, and H. Zidani. Reachability and minimal times for state constrained nonlinear problems without any controllability assumption. SIAM J. Control Optim., 48(7):4292-4316, 2010. 
10. O. Bokanowski, A. Picarelli, and H. Zidani. Dynamic Programming and Error Estimates for Stochastic Control Problems with Maximum Cost. Applied Mathematics and Optimization, 71(1):125-163, 2015.

11. B. Bouchard, R. Elie, and N. Touzi. Stochastic target problems with controlled loss. SIAM J. Control Optim., 48(5):3123-3150, 2009.

12. B. Bouchard and N. Touzi. Weak dynamic programming principle for viscosity solutions. SIAM J. Control Optim., 49(3):948-962, 2011.

13. F. Camilli and M. Falcone. An approximation scheme for the optimal control of diffusion processes. RAIRO Modél. Math. Anal. Numér., 29(1):97-122, 1995.

14. M.G. Crandall, H. Ishii, and P.L. Lions. User's guide to viscosity solutions of second order partial differential equations. Bull. Amer. Math. Soc., 27(1):1-67, 1992.

15. K. Debrabant and E.R. Jakobsen. Semi-Lagrangian schemes for linear and fully non-linear diffusion equations. Math. Comp., 82(283):1433-1462, 2012.

16. M. Falcone, T. Giorgi, and P. Loreti. Level sets of viscosity solutions: Some applications to fronts and rendez-vous problems. SIAM J. Appl. Math., 54:1335-1354, 1994.

17. W. H. Fleming and H. M. Soner. Controlled Markov Processes and Viscosity Solutions, 2nd ed. Springer, 2006.

18. H. Föllmer and P. Leukert. Quantile hedging. SIAM J. of Computational Physics, 3(3):251273, 1999.

19. L. Grüne and A. Picarelli. Zubov's method for controlled diffusions with state constraints. Nonlinear Differential Equations and Applications NoDEA, 22(6):1765-1799, 2015.

20. P.E. Kloeden and E. Platem. Numerical solution of stochastic differential equations. Berlin, New York, Springer-Verlag, 1992.

21. A. Kröner, A. Picarelli, and Z. Zidani. Infinite horizon stochastic optimal control problems with running maximum cost. 2017. Preprint https://hal.archives-ouvertes.fr/hal-01585766.

22. K. Margellos and J. Lygeros. Hamilton-Jacobi formulation for Reach-avoid differential games. IEEE Transactions on Automatic Control, 56:1849-1861, 2011.

23. J.L. Menaldi. Some estimates for finite difference approximations. SIAM J. Control Optim., 27:579-607, 1989.

24. I. Mitchell, A. Bayen, and C. Tomlin. A time-dependent Hamilton-Jacobi formulation of reachable sets for continuous dynamic games. IEEE Trans. Automat. Control, 50:947-957, 2005.

25. S. Osher and A.J. Sethian. Fronts propagating with curvature dependent speed: algorithms on Hamilton-Jacobi formulations. J. Comp. Phys., 79:12-49, 1988.

26. H. Soner and N. Touzi. A stochastic representation for level set equations. Comm in Partial Differential Equations, 27(9-10):2031-2053, 2002.

27. J. Yong and X. Y. Zhou. Stochastic controls, volume 43 of Applications of Mathematics (New York). Springer-Verlag, New York, 1999. Hamiltonian systems and HJB equations. 